Check for updates

Cite this: RSC Adv., 2017, 7, 29656

Received 31st January 2017

Accepted 22nd May 2017

DOI: 10.1039/c7ra01306j

rsc.li/rsc-advances

\section{Selenadiazole derivatives antagonize glucocorticoid-induced osteoblasts cells apoptosis by blocking ROS-mediated signaling, a new anti- osteoporosis strategy}

\author{
Yanxin Du, ${ }^{a}$ Hong Li, ${ }^{b}$ Bolai Chen, ${ }^{a}$ Haogiang Lai, ${ }^{b}$ Xiaoling Li ${ }^{\star c}$ \\ and Tianfeng Chen (D) *b
}

Long-term use of glucocorticoids (GC), especially dexamethasone (Dex), could result in osteoporosis through induction of oxidative stress-mediated apoptosis of osteoblasts and increased differentiation of osteoclasts, finally leading to bone loss. Therefore, searching for new agents that could block Dexinduced cytotoxicity would be a good way to treat osteoporosis. In this study, we show that, synthetic benzo[1,2,5]selenadiazole derivatives (SeDs) could be used as effective inhibitors of Dex-induced osteoblasts apoptosis. This protective effect was correlated with their lipophilicity, cellular uptake and antioxidant activities. Furthermore, mechanistic studies reveal that, treatment of osteoblast cells with Dex resulted in overproduction of intracellular reactive oxygen species (ROS), DNA fragmentation, activation of caspase-3/-9, mitochondria fragmentation, phosphorylation of p53, and activation of MAPKs and AKT pathways. However, pre-treatment of the cells with the synthetic SeDs effectively blocked these intracellular events, which suggest that SeDs could protect osteoblast cells against Dex-induced cell apoptosis via attenuating oxidative stress and downstream signalling pathways. Therefore, this study demonstrates a new therapeutic application of SeDs to antagonize GC-induced osteoporosis.

\section{Introduction}

Glucocorticoid (GC) therapy is widely used in the treatment of chronic inflammatory and autoimmune diseases such as rheumatic, asthma disease, cancer and transplantation. ${ }^{1}$ Nowadays, hundreds of thousands of people receive GC treatment. However, long-term using of GCs could bring severe side effects, such as reduction in bone density. ${ }^{2}$ Bone, a dynamic remodeling tissue, formation is a coupled process including bone resorption by osteoclasts and bone formation by osteoblasts. ${ }^{3}$ Osteocyte, the most abundant bone cell, is the terminally differentiated osteoblasts. ${ }^{4}$ Increasing osteocyte cell death is also considered to be highly associated with accumulation of bone micro damage which is caused by loss of ability to sense micro damage and repair by other bone cells. ${ }^{5}$

GC-induced osteoblasts cells apoptosis is the most common reason of drug-related osteoporosis, and ranks third of osteoporosis following the postmenopausal and senile varieties. ${ }^{6}$ Early studies have showed that GC mainly led to bone loss

${ }^{a}$ Orthopedics Department, Guangdong Provincial Hospital of Traditional Chinese Medicine, 111 Dade Road, Guangzhou 510120, China

${ }^{b}$ Department of Chemistry, Jinan University, Guangzhou 510632, China. E-mail: tchentf@jnu.edu.cn

'Institute of Food Safety and Nutrition, Jinan University, Guangzhou, China. E-mail: tlxlli@jnu.edu.cn through the influence on osteocyte, osteoblasts and osteoclasts. $^{7}$ Excess GCs have prosurvival effect on osteoclasts differentiation, inhibit the activity of osteoblasts, and induce apoptosis of osteoblasts and osteocytes, leading to reduced bone formation and increased bone resorption. ${ }^{8}$ Nowadays, a variety of pharmacological treatments, including bisphosphonates, hormone replacement therapy, and calcium supplementation have been developed for osteoporosis therapy. Fluoride, testosterone, parathyroid hormone, calcitonin and anabolic steroids have been proposed to prevent GC-induced bone lose. ${ }^{9,10}$ However, looking for efficient and low toxicity protectants still kindled great interest of scientists.

Studies have confirmed that many diseases were closely correlated with the generation of reactive oxygen species (ROS). ${ }^{11}$ Multiple cellular molecules could be oxidized by ROS such as unsaturated fatty acid, and the oxidative substances are harmful to human health. Moreover, oxidative stress has been reported to induce osteoblasts apoptosis in MC3T3 osteoblast cells. ${ }^{\mathbf{1 2}}$ Therefore, drugs that possess antioxidants are potential pharmacologic and therapeutic agents. Selenium (Se) is an important trace element, and antioxidant activity is an important biological function. Studies have showed that, inside the biological systems, selenocompounds could effectively remove ROS. ${ }^{13}$ Animal experiments confirmed that, organic selenocompounds showed significant protective effects at the range of 
safe dose against cell damage. ${ }^{14}$ Se could significantly activate SOD, GSH-PX activity, increase the GSH content, and thus enhance the intracellular anti-oxidative capacity. ${ }^{15}$ Allophycocyanin isolated from Se-rich algae could protect the blood cell from AAPH-induced oxidative stress damage by inhibiting ROS generation. ${ }^{10}$ Sodium selenite could reverse the glutamateinduced cytotoxicity associated with ROS production and enhanced oxygen consumption. ${ }^{16}$ In addition, supplementation of Se could bring the gospel to many neurodegenerative diseases by restoring the activity of antioxidant enzymes. ${ }^{17}$ Comparing with inorganic Se, organic selenocompounds show advantages of higher absorptivity and lower toxicity. ${ }^{18}$ Therefore, more and more attentions have been focused on the rational design and discovery of organoselenium in cancer and chemotherapy.

During the past decade, a large number of potent organoselenium compounds, including selenocyanate, selenobetaine and seleno-amino acids, have been designed to achieve greater chemopreventive efficacy. In our previous studies, synthetic selenadiazole derivatives have been found be able to induce cancer cell death through caspase- and p53-dependent apoptosis. ${ }^{19}$ Selenadiazole derivatives could also antagonize hyperglycemia-induced drug resistance in cancer cells through the AMPK-mTORC1 and p53 signaling pathways. ${ }^{20,21}$ Another series of selenadiazole derivatives were also identified as thioredoxin reductase inhibitors that induced cancer cell apoptosis. ${ }^{22,23}$ Recently, a novel selenium-containing ruthenium complex was found to enhance radiation-induced DNA damage, which led to G2/M arrest and apoptosis in cancer cells by activating ROS-mediated pathways. ${ }^{24}$ All of our previous works has suggested that Se-containing compounds exhibited potency in cancer therapy. However, little information on their ability to antagonize GC-induced osteoporosis is available. Interestingly, herein a series of benzo[1,2,5] selenadiazole derivatives have been screened and identified as effective inhibitors of Dexinduced osteoblasts apoptosis. This protective effect was correlated with their lipophilicity, cellular uptake and antioxidant activities. Furthermore, we demonstrated that, treatment of MC3T3 osteoblast cells with Dex resulted in overproduction of intracellular reactive oxygen species (ROS), DNA fragmentation, activation of caspase-3/-9, mitochondria fragmentation, phosphorylation of p53, and activation of MAPKs and AKT pathways. However, pre-treatment of the cells with the synthetic SeDs effectively blocked these intracellular events induced by Dex, which suggest that SeDs could protect osteoblast cells against Dex-induced cell apoptosis via attenuating oxidative stress and downstream signalling pathways. Therefore, this study demonstrates a new therapeutic application of SeDs to antagonize GC-induced osteoporosis.

\section{Results and discussion}

Relationship among the lipophilicity, cellular uptake and antioxidant activities of SeDs

Our previous work has showed that organoselenium compounds with selenadiazole moiety had good anticancer and antioxidantactivities. ${ }^{14,20-23,25-32}$ Therefore we tried to make some decoration on the benzo[1,2,5]selenadiazole by introduction of various alcohol to enhance the solubility and antioxidant activities. A series of 5-alkylbenzenecarboxyl[c][1,2,5]selenadiazoles with different alkyls, such as methyl, ethyl, propyl, isopropyl, butyl groups, were synthesized according to previous described..$^{21,33}$ These SeDs were expected to antagonize Dexinduced apoptosis and anti-osteoporosis activities in MC3T3 osteoblast cells (Fig. 1). Glucocorticoid receptor (GR) transactivation induced by Glucocorticoid (GC) has been confirmed to highly correlate with GC-induced osteoblasts cells apoptosis. In order to investigate whether selenadiazole derivatives (SeDs) have anti-glucocorticoid receptor (GR) effect, we firstly carried out experiments to examine the inhibition activities of SeDs on GR transactivation. As shown in Fig. 2A, the SeDs alone could effectively inhibit the expression of GR. Especially, IBSe demonstrated the highest inhibitory effects. Furthermore, we tried to test the effects of Dex and the inhibitory effect of IBSe. As shown in Fig. 2B, Dex alone significantly increased the expression level of GR. In contrast, the combined treatment with IBSe effectively reversed this effect. Taken together, these results suggest that, GR may be involved in the antagonizing activity of SeDs against Dex-induced osteoblasts cells apoptosis. Evidence have revealed the correlationship between oxidative stress and osteoblasts apoptosis, ${ }^{12}$ therefore, these SeDs were then screened for their antioxidant and anti-osteoporosis activities. As we all known, the lipophilicity of a compound is well known to have significant influence on its biological activity. ${ }^{34}$ Therefore, we have detected the relationship between lipo-hydro partition coefficient $(\log P)$ and cellular uptake of the synthetic compounds. As shown in Fig. 3A, the increase in the length and molecular weight of alkyl groups didn't linearly increased the lipophilicity of the synthetic selenadiazole derivatives, with higher $\log P$ values detected in EBSe and PBSe. Consistently, EBSe and PBSe demonstrated significant higher cellular uptake in MC3T3 cells and the five compounds were well lipophilic. These results demonstrate the positive relationship between the lipophilicity and cellular uptake of the synthetic compounds. We then examined the antioxidant activities of SeDs by using ABTS assay. As showed in Fig. 3B-F, all of the compounds could effectively scavenge the free radicals in a time-dependent manner, which demonstrating the potent

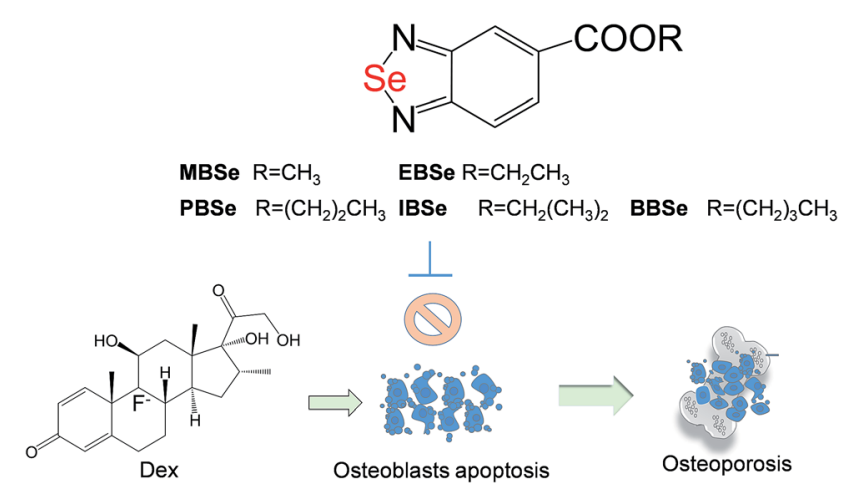

Fig. 1 Antagonization of Dex-induced apoptosis by selenadiazole derivatives. 


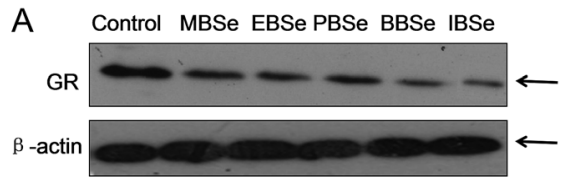

$\mathrm{B}$

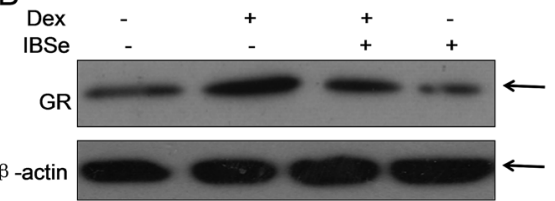

Fig. 2 GR expression inhibition of selenadiazole derivatives. (A) SeDs inhibited GR transactivation. (B) IBSe reversed GR transactivation induced by DeX.
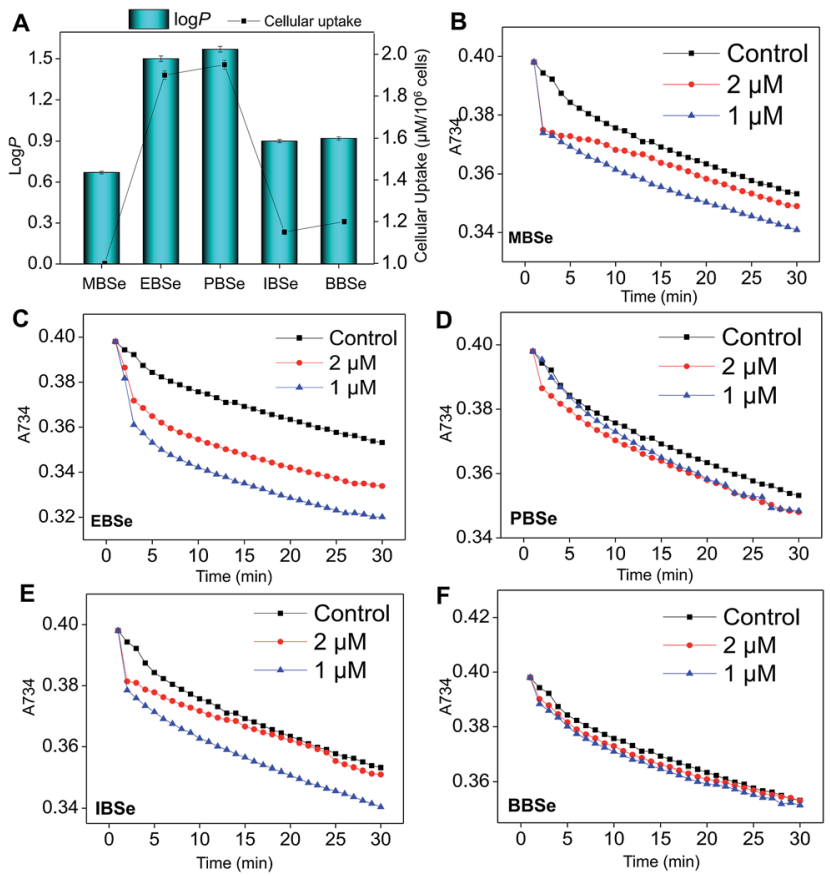

Fig. 3 Lipophilicity, cellular uptake and antioxidant activities of selenadiazole derivatives. (A) The lipophilicity and cellular uptake of SeDs. The intracellular Se concentration in cells exposed to $0.2 \mu \mathrm{M}$ SeDs was determined by ICP-AES analysis. Antioxidant activities of MBSe (B), EBSe (C), PBSe (D), IBSe (E) and BBSe (F) by ABTS assay.

antioxidant activity of these compounds. It is well known that TrxR is homodimeric selenoenzyme and plays crucial role in antioxidant defense through regulating the activation and reduction of Trx as well as other oxidized cellular components by counteracting excess reactive oxygen species (ROS). Therefore, in order to further investigate whether SeDs antioxidant activity was due to the upregulation of selenoproteins. We next examined the effects of IBSe and Dex on the activity of selenoenzyme, TrxR. As shown in Fig. 4, Dex alone significantly inhibited the activity, IBSe effectively elevated TrxR activity. In contrast, the combined treatment with IBSe effectively reversed this effect. Taken together, these results suggest that, IBSe may antagonize Dex-induced osteoblasts cells apoptosis by

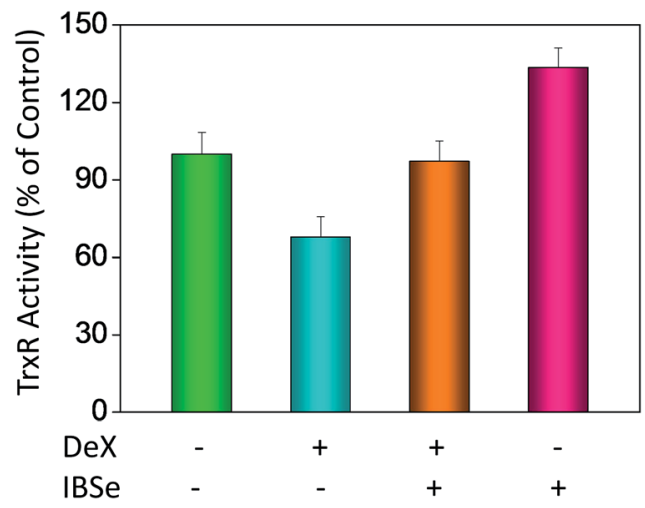

Fig. 4 Selenadiazole derivatives reverse Dex-inhibited intracellular TrxR activity in MC3T3 cells.

increasing selenoenzyme activity which supports the application potential of the compounds against oxidative stress-related diseases.

\section{SeDs reduce Dex-induced cytotoxicity through blocking cell apoptosis}

Studies were also carried out to evaluate the protective effects of the synthetic compounds on Dex-induced cytotoxicity in MC3T3 osteoblast cells. As shown in Fig. 5A, treatment of the cells with Dex at 0.1 to $10 \mu \mathrm{M}$ for $48 \mathrm{~h}$ reduced the cell viability in a dosedependent manner. However, pre-treatments of the cells with all the SeDs significantly increased the cell viability (Fig. 5B-F), while the compounds alone showed slightly inhibition effects in the cell growth. Among them, IBSe exhibited better protective effect on Dex-induced cytotoxicity, which may be due to its strong antioxidant activity. Studies were also conducted to examine the action mechanisms of Dex and the synthetic SeDs. Apoptosis is a highly programmed cell death, which has been identified as the major mode of cell death induced by Dex in MC3T3 osteoblast cells. ${ }^{35}$ Therefore, in this study, firstly, we have analyzed the effects of Dex and selenadiazole derivatives on the cell cycle distribution by flow cytometric analysis. As shown in Fig. 5G, Dex $(10 \mu \mathrm{M})$ caused $15.7 \%$ of the cell apoptosis and $22.61 \%$ G0/G1 cycle arrest. However pre-treated the cells with MBSe, EBSe, PBSe, IBSe and BBSe $(0.2 \mu \mathrm{M})$ for $2 \mathrm{~h}$, obviously reduced the apoptosis from $15.7 \%$ to $6 \%, 8.8 \%$, $7.4 \%, 2.4 \%, 6.3 \%$ and $\mathrm{G0} / \mathrm{G} 1$ cycle arrest from $22.6 \%$ to $15.06 \%$, $12.7 \%, 11.68 \%, 15.05 \%, 15.16 \%$. Among them, preventive effect of IBSe was the most obvious. Alkaline phosphatase (ALP) is the earliest marker of osteoblasts MC3T3 osteoblast cells differentiation. ${ }^{36,37}$ Then we detected the activity of ALP to assess the protective effect of the five SeDs on osteoblasts differentiation. As shown in Fig. 6A, incubation of cells with Dex $(0.1,1,10 \mu \mathrm{M})$ for $72 \mathrm{~h}$ visibly reduced the activity of ALP. However, pretreatment of IBSe for $2 \mathrm{~h}$ significantly abolished these effects induced by Dex. Additionally, we found that MBSe, EBSe, PBSe and BBSe displayed neglectable effects towards ALP activity (Fig. 6B-E) Taken together, these results suggest that IBSe is capable of reversing the apoptosis effects induced by Dex. 

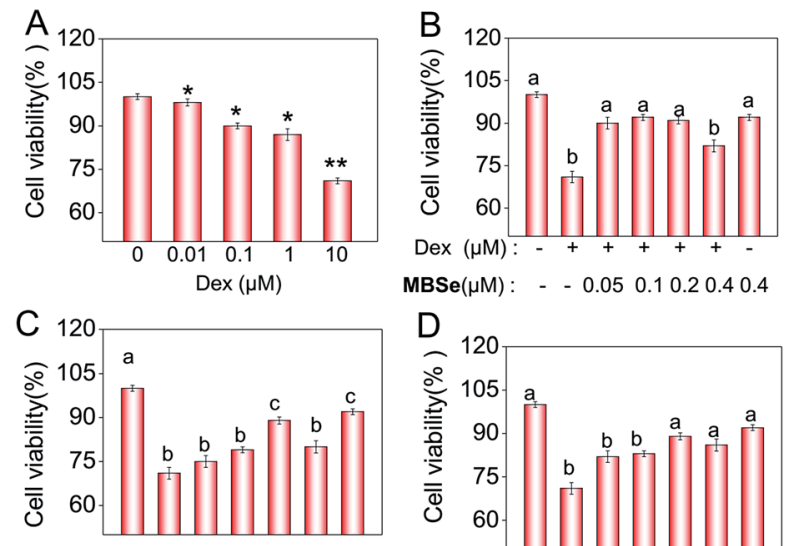

$\operatorname{Dex}(\mu \mathrm{M}):-+++++$
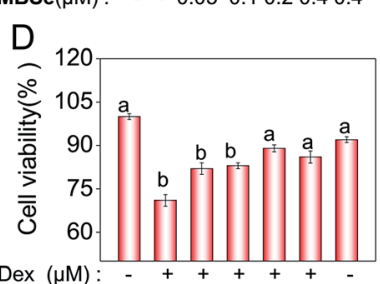

EBSe $(\mu \mathrm{M})$ : - $\quad-0.050 .10 .20 .40 .4$ PBSe $(\mu \mathrm{M})$ : - $\quad-0.050 .10 .20 .40 .4$
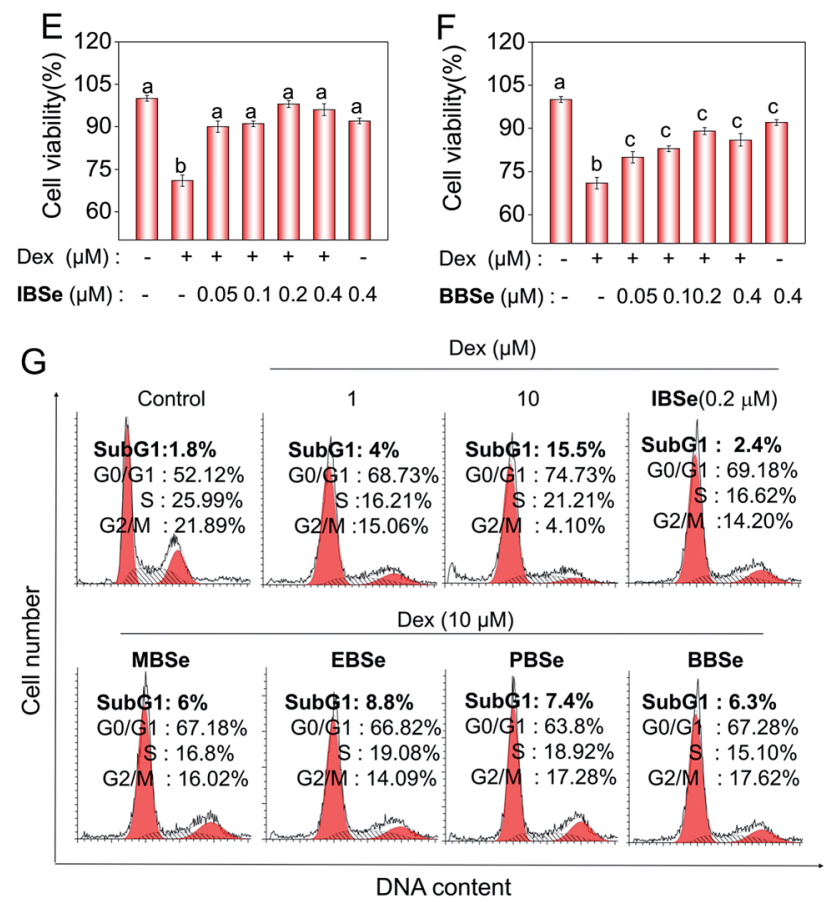

Fig. 5 SeDs reverse the apoptosis-inducing effects of Dex. Cytotoxicity of Dex and SeD toward MC3T3 cells (A-F). (G) SeDs attenuate Dex-induced apoptosis in MC3T3 cells. Quantitative analysis of apoptotic cell death induced by SeDs and Dex were analyzed by flow cytometric analysis. All data here are expressed as means \pm SD of triplicates. Bars with different characters $(\mathrm{a}-\mathrm{c})$ are statistically different at $P<0.05$ level. $* P<0.05$ vs. untreated control. $* * P<0.01$ vs. untreated control.

\section{IBSe alleviates Dex-induced apoptosis through inhibition of caspase activation and mitochondrial dysfunction}

Caspase, a family of cysteine acid proteases, is known as important mediators of cell apoptosis and contributed to the apoptotic morphology through cleavage of various cellular substrates..$^{38}$ Generally upon stimulation, apoptosis occurs through mitochondria-mediated (intrinsic) pathway and death receptor-mediated (extrinsic) pathway. ${ }^{39,40}$ These two pathways exert their roles by activating various caspases. Therefore, caspases activation is an important indicator of apoptosis.
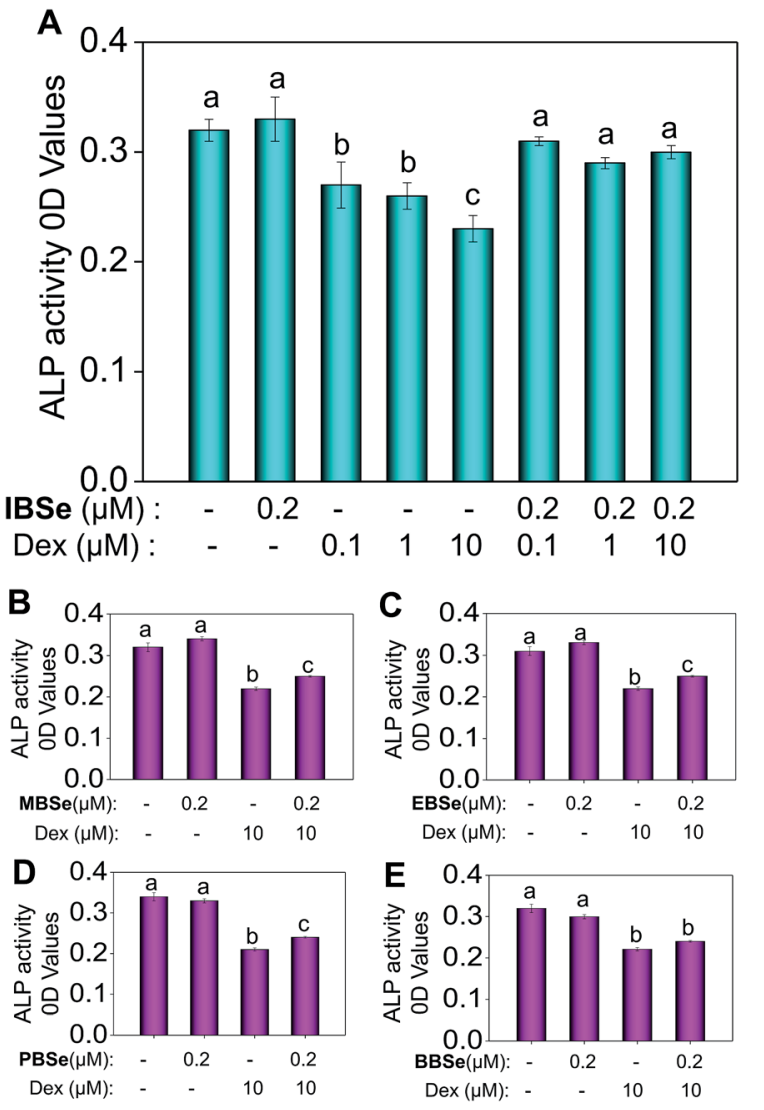

Fig. 6 Effects of DEX and SeDs on the ALP activity of osteoblast. Osteoblast was used to be treated with Dex or SeDs with different concentration, (A) IBSe, (B) MBSe, (C) EBSe, (D) PBSe, (E) BBSe. Bars with different characters $(a-C)$ are statistically different at $P<0.05$ level.

Consequently, we conducted fluorometric assay to detect whether the activation of caspase-3/-8/-9 was involved in this process. As depicted in Fig. 7B, IBSe induced slightly upregulation of actived caspase-3/-8/-9, while Dex triggered significant activation of caspase-3/-9 in MC3T3 cells. In contrast, pretreatment of the cells with $0.2 \mu \mathrm{M}$ IBSe remarkably restrained the caspases activation induced by Dex, which indicated the protective effects of IBSe against the caspases activation inducing capacity of Dex towards MC3T3 osteoblast cells. Both the extrinsic and intrinsic apoptotic signals have been shown to integrated in mitochondria. And deleption of mitochondria membrane potential $\left(\Delta \psi_{\mathrm{m}}\right)$ is considered to be a highly relevant event upon apoptosis stimulation. ${ }^{41}$ Therefore, we conducted the following study, cells were pretreated with IBSe for $2 \mathrm{~h}$, then co-cultured with Dex for $48 \mathrm{~h}$, after that, cells were stained with the mitochondria-selective JC-1 dye and analyzed by flow cytometry. The red fluorescence represented normal mitochondrial membranes potential, while intensity of green fluorescence represented the cells that lost mitochondrial membrane potential as a result of the JC- 1 monomers. As shown in Fig. 7A, Dex treatment dose-dependently induced deleption of $\Delta \psi_{\mathrm{m}}$ from $5.42 \%$ to $22.3 \%, 36.8 \%$ and $76.2 \%$. However, pretreatment of $0.2 \mu \mathrm{M}$ IBSe effectively blocked the mitochondria depolarization induced by Dex, as demonstrated by the decreased green fluorescence intensity. 
A

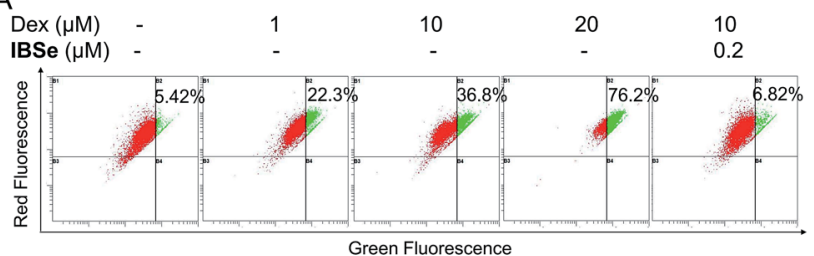

$\mathrm{B}$

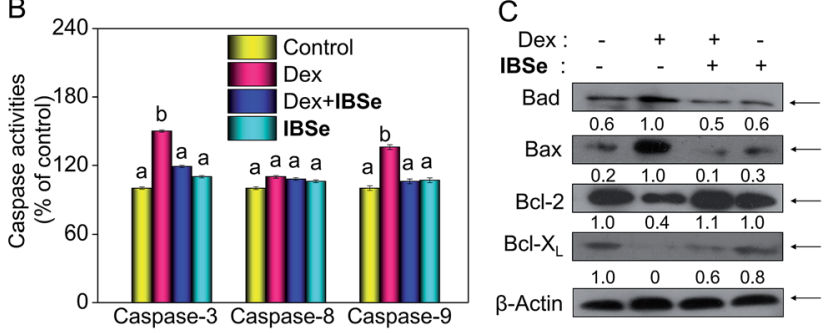

Fig. 7 IBS reduces caspase mediated mitochondrial membrane potential induced by Dex. (A) Cells were pre-treated with IBSe for $2 \mathrm{~h}$, then co-cultured with Dex for another $48 \mathrm{~h}$ and analyzed by JC-1 flow cytometry. (B) Caspase activities were measured by specific fluorescent substrates for caspase-3/8/9. (C) The expression of Bcl-2 protein family. Significant difference between treatment and control groups in indicated at $P<0.05\left(^{*}\right)$ and $P<0.01(* *)$ levels.

The Bcl-2 family members have been known as the key regulators of mitochondrial permeability. Here we measured the expression level of Bcl-2 family members by immunoblotting assay. Dex incubation up-regulated the expression of proapoptosis protein, such as Bax and Bad, but the downregulated the expression level of pro-survival proteins, such as $\mathrm{Bcl}-2$ and $\mathrm{Bcl}-\mathrm{x}_{\mathrm{L}}$. However, noticeable reversed effects were observed in Bcl-2 family proteins expression triggered by Dex after the pretreatment with IBSe for $2 \mathrm{~h}$ (Fig. 7C). Together, these results confirm that IBSe protects MC3T3 cells against Dex-induced apoptosis through inhibition of caspases activation and mitochondria dysfunction.

\section{IBSe prevents Dex-induced mitochondria fragmentation}

Since IBSe obtains protective effects against Dex destruction on mitochondria, we next conducted morphological studies of mitochondria by using Mito Tracker Green CMXRos as a marker through employing fluorescence microscopy. ${ }^{42}$ As illustrated in Fig. 8, $0.2 \mu \mathrm{M}$ IBSe incubation induced neglectable disruption in mitochondria structure, while treatment of the MC3T3 cells with $10 \mu \mathrm{M}$ Dex resulted in remarkable fragmentation of mitochondria compared with the control groups. Significant reduction in these changes was observed in groups that pretreated with IBSe for $2 \mathrm{~h}$ and then cotreated with Dex for $48 \mathrm{~h}$. The morphological improvements demonstrated that IBSe could restrain the Dex-induced damage to mitochondria in MC3T3 cells.

\section{IBSe decreases Dex-induced p53 phosphorylation through eliminating ROS accumulation in MC3T3 cells}

ROS, including superoxide, hydrogen peroxide, and hydroxyl radical, accumulation has been confirmed to be the results of

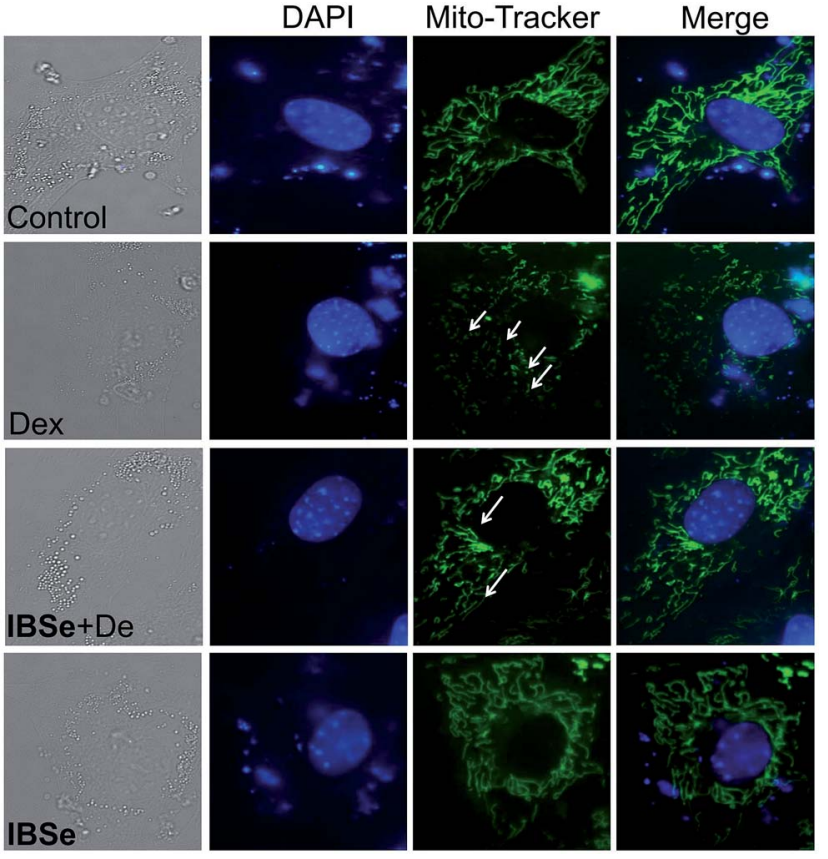

Fig. 8 IBSe reduces mitochondria fragmentation induced by Dex. Cells were pre-treated with or without IBSe $(0.2 \mu \mathrm{M})$ for $2 \mathrm{~h}$, then cultured with Dex $(10 \mu \mathrm{M})$ for $48 \mathrm{~h}$, after that cells were Stained by Green Mito-Tracker for $2 \mathrm{~h}$ and stained by DAPI for $30 \mathrm{~min}$.

mitochondria dysfunction. And ROS generation is shown to be the important mechanisms of osteoblasts apoptosis induced by high dose GCs. Thus, we examined whether attenuation of ROS was implicated in IBSe protective effects towards Dex-induced apoptosis by using a specific probe DHE. ${ }^{43}$ As shown in Fig. 9A, significant increase of ROS was observed in cells that treated with Dex $(10 \mu \mathrm{M})$, which induced the apoptosis of MC3T3 osteoblast cells. However, pretreatment of the cells with MBSe, EBSe, PBSe, IBSe and BBSe significantly reduced the Dexinduced ROS generation a time-dependent manner, Moreover, IBSe was found to potently inhibited Dex triggered ROS generation in osteoblast cells (Fig. 9B). Evidences have demonstrated that overproduction of ROS may result in DNA damage and thus activated p53, which takes part in apoptosis modulation through regulating various downstream proteins expression, such as p21, Bax, Bcl-2 and Bcl-XL. Therefore, p53 and p21 proteins expression level was evaluated by western blotting assay to confirm the protective effects. The results revealed that IBSe strongly blocked Dex-induced p53 activation and p21 expression (Fig. 9C). Together, these results demonstrate that IBSe effectively inhibits Dex-induced p53 phosphorylation through decreasing ROS accumulation in MC3T3 cells.

\section{IBSe attenuates Dex-induced apoptosis through regulating AKT and MAPKs pathways}

ERK and Akt signaling pathways have been confirmed to play important role in cell apoptosis and cell proliferation. ${ }^{44}$ Akt enhances the survival of cells by inhibiting the expression of pro-apoptotic proteins, such as caspase-9 and p53. ${ }^{45}$ Similar to 
A

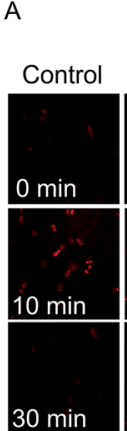

$\mathrm{B}$

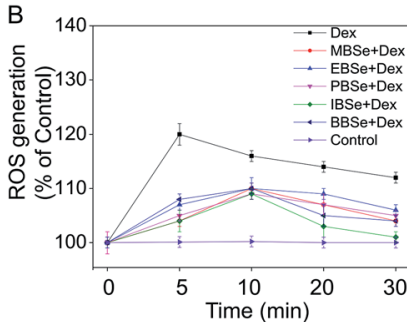

Fig. 9 IBSe prevents Dex induced ROS generation in MC3T3 cells. (A) Cells pretreatment with SeDs for $2 \mathrm{~h}$ and then co-incubated with Dex. After treatment cells were stained with DHE-DA for $30 \mathrm{~min}$, and then imaged under the fluorescence microscope (magnification, 10x). (B) Dex and SeDs induced ROS accumulation in MC3T3 cells. Cells were treated with indicated concentrations of drugs for various periods of time. The levels of intracellular ROS were determined by DHE-DA assay. (C) Protein expression of p53 and p21.

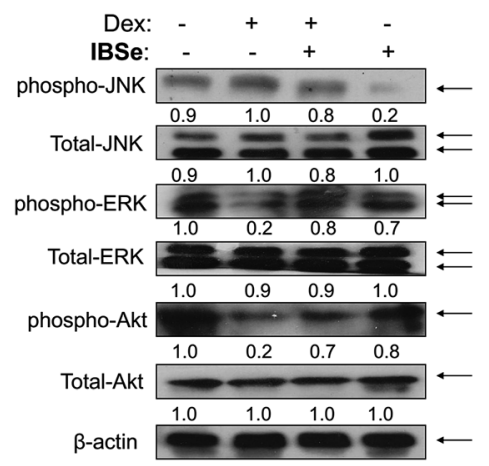

Fig. 10 IBSe and Dex impact on Akt and MAPK signaling pathway. Cells were pre-treated with IBSe $(0.2 \mu \mathrm{M})$ for $2 \mathrm{~h}$, then cultured with Dex $(10 \mu \mathrm{M})$ for $48 \mathrm{~h}$. The phosphorylation status and expression levels of Akt and MAPK after treatment of IBSe and Dex. Equal protein loading was confirmed by Western analysis of $\beta$-actin in the protein extracts.

Akt, ERK are involved in many kinds of cellular processes, including apoptosis, cell proliferation and differentiation. ${ }^{46}$ It could also prevent cell apoptosis by blocking the activation of caspase. ${ }^{47}$ Contrary to Akt and ERK, MAPK has been reported to induce cell apoptosis in response to external stimuli. ${ }^{48}$ Oxidative stress has been identified as a key modulator of MAPK signaling cascades. ${ }^{49}$ In this study, ROS overproduction induced by Dex up-regulated the phosphorylation of JNK, and down-regulated the phosphorylation of ERK and AKT (Fig. 10). In contrast, pretreatment of the cells with IBSe effectively reversed these effects induced by Dex. These results indicate that, SeDs

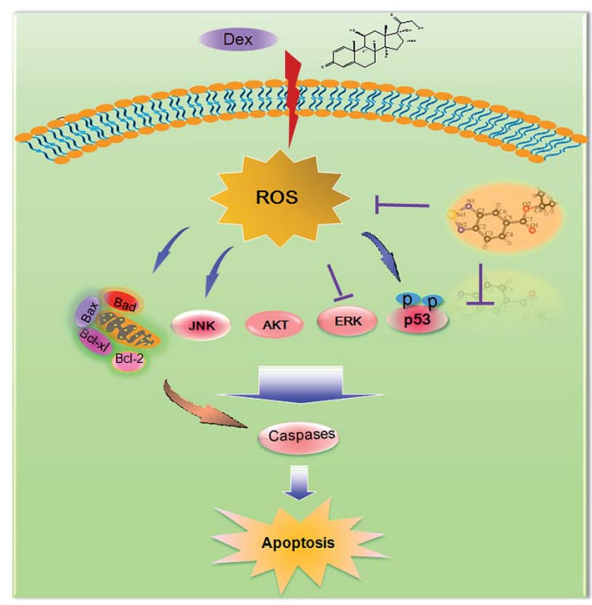

Fig. 11 The proposed protective signaling pathway of IBSe againsts Dex-induced apoptosis in MC3T3 cells.

prevented Dex-induced osteoblast cells apoptosis by suppression of pro-apoptotic JNK kinase and activation of pro-survival ERK and AKT kinases. Based on the above results, we proposed a signaling network for the antagonism action of selenadiazole derivative on Dex-induced apoptosis (Fig. 11).

\section{Conclusions}

In this study, a series of benzo[1,2,5]selenadiazole derivatives has been screened and identified as effective inhibitors of Dexinduced osteoblasts apoptosis. The protective effects were correlated with their lipophilicity, cellular uptake and antioxidant activities against Dex-induced oxidative damage. Further mechanic studies suggest that the synthetic selenadiazole derivatives displayed noticeable protective effects against Dexinduced cell apoptosis through attenuating oxidative damage mediated p53 phosphorylation and regulating AKT and MAPKs signaling pathways. Taken together, this study demonstrates a new therapeutic application of SeDs to antagonize GCinduced osteoporosis.

\section{Experimental section}

\section{Materials}

Dexamethasones (Dex), Propidium Iodide (PI), thiazolyl blue tetrazolium bromide (MTT), solid JC-1, dihydroethidium (DHE), bicinchoninic acid (BCA) kit were purchased from Sigma (sigma-Aldrich, St. Louis, MO). All antibodies were purchased from Cell Signaling Technology (Beverly, MA). Caspase-8, caspase-9 and caspase-3 substrates were purchased from Calbiochem.

\section{Synthesis and characterization of selenadiazole derivatives}

The synthesis of SeDs was conducted according to our previous methods. $^{20,21}$ 


\section{Cell culture, drugs treatment and measurement of cell} viability

MC3T3 cell line was obtained from American Type Culture Collection (ATCC, Manassas, Virginia). The cells were cultured with $\alpha$-MEM medium, which supplemented with fetal bovine serum (10\%), penicillin (100 units per $\mathrm{mL}$ ) and streptomycin (50 units per $\mathrm{mL})$ at $37{ }^{\circ} \mathrm{C}$ in a humidified $\left(5 \% \mathrm{CO}_{2}\right)$ atmosphere. The cells were seeded in culture plates for $24 \mathrm{~h}$, and then pretreated with five benzo[1,2,5]selenadiazole derivatives for $2 \mathrm{~h}$ and co-incubated with Dex for another $48 \mathrm{~h}$ to examine the synergistic effects on MC3T3 osteoblast cells. Cell viability was determined by using MTT assay as previously described. ${ }^{\mathbf{5 0}}$

\section{Flow cytometric analysis}

The cell cycle distribution was analyzed by flow cytometry as previously described. ${ }^{50}$ Apoptotic cells were measured by quantifying the sub-G1 peak. For each experiment, 10000 events per sample were recorded.

\section{Determination of lipophilicity and cellular uptake}

The lipophilicity of MBSe, EBSe, PBSe, IBSe and BBSe was determined by using the "shake-flask" method previously reported. ${ }^{51}$ The content of Se in each phase was determined by ICP-MS analysis. $\log P$ was calculated as the logarithmic ratio of the concentrations of the drug in the $n$-octanol phases and aqueous phases. The intracellular Se concentration was determined by ICP-MS method, as previously described. ${ }^{52}$ MC3T3 osteoblast cells were harvested after incubation with $0.2 \mu \mathrm{M}$ five compounds for $2 \mathrm{~h}$, and then washed with PBS buffer for three times. Cells were digested for $3 \mathrm{~h}$ in mixture of perchloric acid and nitric acid at the ratio of $3: 1$. Then digestive was volume to $5 \mathrm{~mL}$ with pure water.

\section{Caspase activity assay}

The specific caspase substrates (Ac-DEVD-AMC for caspase-3, Ac-IETD-AMC for caspase-8 and Ac-LEHD-AMC for caspase-9) were placed in 96-well plates and then total cell lysates $(50 \mu \mathrm{g}$ per well) were added. ${ }^{50}$ Plates were incubated for $2 \mathrm{~h}$ at $37^{\circ} \mathrm{C}$ in darkness and the caspase activity was determined by fluorescence intensity with the excitation and emission wavelengths set at 380 and $460 \mathrm{~nm}$ respectively.

\section{Evaluation of mitochondrial membrane potential $\Delta \psi_{\mathrm{m}}$}

After treatment, cells were in incubated with $0.5 \mathrm{~mL}$ of PBS buffer containing $10 \mu \mathrm{g}$ per $\mathrm{mL}$ of JC-1 for $20 \mathrm{~min}$. Stained cells were immediately centrifuged to remove the supernatant, and added $0.5 \mathrm{~mL}$ PBS, at last analyzed by flow cytometry. ${ }^{53}$ The percentage of green fluorescence was used to represent the cells that lost $\Delta \psi_{\mathrm{m}}$.

\section{Mitochondria fragmentation}

The cells were pretreated with IBSe for $2 \mathrm{~h}$, and then co-cultured with Dex for another $48 \mathrm{~h}$; cell mitochondria were stained with 100 nM MitoTracker Green CMXRos for 90 min and nucleuses stained with $1 \mu \mathrm{g}$ per mL DAPI for another $20 \mathrm{~min}$. After that, the cells were washed with PBS twice, and cultured in fresh medium on a thermo-cell culture FCS2 chamber of Carl Zeiss Cell Observer (Jena, Germany). Cell images were captured with a monochromatic CoolSNAPFX camera (Roper Scientific, USA) and analyzed by using Axio Vision 4.2 software (Carl Zeiss).

\section{Measurement of intracellular reactive oxygen species (ROS) generation}

The intracellular ROS generation was detected by DHE probe fluorescence assay. ${ }^{54}$ The DHE fluorescence intensity of treated cells was expressed as percentage of control (as 100\%)

\section{ABTS free radical scavenging assay}

ABTS free radical scavenging activity of MBSe, EBSe, PBSe, IBSe and BBSe were measured as previously described. ${ }^{38}$ To determine the antioxidant activity, $1 \mathrm{~mL}$ ABTS reagent and $50 \mu \mathrm{L}$ of sample or positive controls was mixed. Absorbance was detected at $734 \mathrm{~nm}$ for $2 \mathrm{~h}$.

\section{Alkaline phosphatase (ALP) activity assay}

The induction of ALP is an unequivocal marker for bone cell differentiation. To measure the ALP activity, cells were seeded in 48 -well plate at $1.5 \times 10^{4}$ per well. After $24 \mathrm{~h}$ cells were pretreated with/without MBSe, EBSe, PBSe, IBSe and BBSe $(0.2 \mu \mathrm{M})$, and then cultured with Dex $(0.1,1,10 \mu \mathrm{M})$. After culture for 3 days, the medium was collected. $20 \mu \mathrm{L}$ medium were taken on the 96-wells, then $100 \mu \mathrm{L}$ reaction liquid (0.5 M Tris, $10 \mathrm{mM} p$ nitro phenyl phosphate, $2 \mathrm{mM} \mathrm{MgCl}_{2}, 1 \mathrm{mM} \mathrm{ZnCl}_{2}, \mathrm{pH}=9.0$ ) were added. After incubation for $30 \mathrm{~min}$ at $37{ }^{\circ} \mathrm{C}$ in the incubator, the reaction was terminated by $1 \mathrm{M} \mathrm{NaOH}(80 \mu \mathrm{L})$. The absorbance at $409 \mathrm{~nm}$ was measured using a spectrophotometric plate reader.

\section{Determination of TrxR inhibition}

The inhibition of TrxR in MC3T3 cells induced by SeDs at different concentrations within $1 \mathrm{~h}$ was determined by the thioredoxin reductase assay kit (Cayman) as previously described. ${ }^{25}$

\section{Western blot analysis}

The effects of SeDs and Dex on the proteins expression level of glucocorticoid receptor (GR) and other different signaling pathways were detected by Western blot analysis. ${ }^{55} \beta$-actin was used to confirm the equal loading and transfer of proteins.

\section{Statistical analysis}

Experiments were carried out at least in triplicate and repeated three times. All data were expressed as mean \pm S.D. Statistical analysis was performed using SPSS statistical package (SPSS 13.0 for Windows; SPSS, Inc. Chicago, IL). The difference between two groups was analyzed by two-tailed Student's $t$-test. The difference between three or more groups was analyzed by one-way analysis of variance multiple comparisons. Differences with $P<0.05$ or $P<0.01$ was considered statistically significant. 


\section{Acknowledgements}

This work was supported by Natural Science Foundation of China (21371076), National High-level personnel of special support program, Science Foundation for Distinguished Young Scholars of Guangdong Province (S2013050014667), YangFan Innovative \& Entrepreneurial Research Team Project (201312H05), Guangdong Special Support Program and Guangdong Frontier Key Technological Innovation Special Funds (2014B050505012) and Fundamental Research Funds for the Central Universities.

\section{Notes and references}

1 H. Xie, S.-Y. Tang, H. Li, X.-H. Luo, L.-Q. Yuan, D. Wang and E.-Y. Liao, Amino Acids, 2008, 35, 419-423.

2 D. Patschan, K. Loddenkemper and F. Buttgereit, Bone, 2001, 29, 498-505.

3 D. Zhang, J. Zhang, C. Fong, X. Yao and M. Yang, Biochimie, 2012, 94, 2514-2522.

4 L. F. Bonewald and M. L. Johnson, Bone, 2008, 42, 606-615. 5 S. C. Manolagas, J. Bone Miner. Res., 2000, 15, 1001-1005.

6 G. Gu, T. Hentunen, M. Nars, P. Härkönen and H. Väänänen, Apoptosis, 2005, 10, 583-595.

7 S. C. Manolagas, Endocr. Rev., 2000, 21, 115-137.

8 P. Moutsatsou, E. Kassi and A. G. Papavassiliou, Trends Mol. Med., 2012, 18, 348-359.

9 J.-F. Zhang, G. Li, C.-L. Meng, Q. Dong, C.-Y. Chan, M.-L. He, P.-C. Leung, Y.-O. Zhang and H.-F. Kung, Phytomedicine, 2009, 16, 521-529.

10 H. Zhang, T. Chen, J. Jiang, Y.-S. Wong, F. Yang and W. Zheng, J. Agric. Food Chem., 2011, 59, 8683-8690.

11 H. Kawagishi and T. Finkel, Nat. Med., 2014, 20, 711-713.

12 M. Arai, Y. Shibata, K. Pugdee, Y. Abiko and Y. Ogata, IUBMB Life, 2007, 59, 27-33.

13 G. K. Azad and R. S. Tomar, Mol. Biol. Rep., 2014, 41, 48654879.

14 Y.-F. Li, Z. Dong, C. Chen, B. Li, Y. Gao, L. Qu, T. Wang, X. Fu, Y. Zhao and Z. Chai, Environ. Sci. Technol., 2012, 46, 11313-11318.

15 E. Song, C. Su, J. Fu, X. Xia, S. Yang, C. Xiao, B. Lu, H. Chen, Z. Sun and S. Wu, Life Sci., 2014, 109, 37-43.

16 S. Kumari, S. L. Mehta and P. A. Li, PLoS One, 2012, 7, e39382.

17 K. Venardos, G. Harrison, J. Headrick and A. Perkins, J. Trace Elem. Med. Biol., 2004, 18, 81-88.

18 B. Laffon, V. Valdiglesias, E. Pásaro and J. Méndez, Biol. Trace Elem. Res., 2010, 133, 12-19.

19 T. Chen and Y.-S. Wong, Int. J. Biochem. Cell Biol., 2009, 41, 666-676.

20 Y. Yang, S. Deng, Q. Zeng, W. Hu and T. Chen, Dalton Trans., 2016, 45, 18465-18475.

21 Y. Liu, Y. Luo, X. Li, W. Zheng and T. Chen, Chem. -Asian J., 2015, 10, 642-652.

22 Q. Xie, Y. L. Zhou, G. Q. Lan, L. Y. Yang, W. J. Zheng, Y. W. Liang and T. F. Chen, Biochem. Biophys. Res. Commun., 2014, 449, 88-93.
23 Y. Liu, Y. Luo, X. Li, W. Zheng and T. Chen, Chem. -Asian J., 2015, 10, 642-652.

24 S. Movva, L. Rodriguez, H. Arias-Pulido and C. Verschraegen, Cancer, 2009, 115, 3166-3180.

25 L. He, S. Ji, H. Lai and T. Chen, J. Mater. Chem. B, 2015, 3, 8383-8393.

26 T. Chen, Y. S. Wong, W. Zheng and J. Liu, Chem.-Biol. Interact., 2009, 180, 54-60.

27 T. Chen and Y. S. Wong, J. Agric. Food Chem., 2008, 56, 10574-10581.

28 T. Chen, W. Zheng, Y. S. Wong and F. Yang, J. Steroid Biochem., 2008, 62, 77-84.

29 Q. Xie, Y. Zhou, G. Lan, L. Yang, W. Zheng, Y. Liang and T. Chen, Biochem. Biophys. Res. Commun., 2014, 449, 88-93.

30 Y. W. Liang, J. Zheng, X. Li, W. Zheng and T. Chen, Eur. J. Med. Chem., 2014, 84, 335-342.

31 Q. Xie, L. He, H. Lai, W. Zheng and T. Chen, RSC Adv., 2014, 4, 34210-34216.

32 M. Zhou, S. Ji, Z. Wu, Y. Li, W. Zheng, H. Zhou and T. Chen, Eur. J. Med. Chem., 2015, 96, 92-97.

33 Y. Yang, S. Deng, Q. Zeng, W. Hu and T. Chen, Dalton Trans., 2016, 45, 18465-18475.

34 L. Messori, F. Scaletti, L. Massai, M. A. Cinellu, C. Gabbiani, A. Vergara and A. Merlino, Chem. Commun., 2013, 49, 1010010102.

35 L. He, Y. Huang, H. Zhu, G. Pang, W. Zheng, Y. S. Wong and T. Chen, Adv. Funct. Mater., 2014, 24(19), 2754-2763.

36 Z.-S. Xu, X.-Y. Wang, D.-M. Xiao, L.-F. Hu, M. Lu, Z.-Y. Wu and J.-S. Bian, Free Radical Biol. Med., 2011, 50, 1314-1323.

37 L. Jin, Y. Nonaka, S. Miyakawa, M. Fujiwara and Y. Nakamura, Mol. Ther., 2016, 24, 1974-1986.

38 T. Chen and Y.-S. Wong, J. Agric. Food Chem., 2008, 56, 43524358.

39 M. R. de Oliveira, Toxicol. Lett., 2016, 258, 185-191.

40 W.-X. Zong, J. D. Rabinowitz and E. White, Mol. Cell, 2016, 61, 667-676.

41 S. Pierotti, L. Gandini, A. Lenzi and A. M. Isidori, J. Steroid Biochem., 2008, 108, 292-299.

42 M. G. Scioli, V. Cervelli, G. Arcuri, P. Gentile, E. Doldo, A. Bielli, E. Bonanno and A. Orlandi, J. Cell. Physiol., 2014, 229, 2077-2087.

43 M. Almeida, L. Han, E. Ambrogini, R. S. Weinstein and S. C. Manolagas, J. Biol. Chem., 2011, 286, 44326-44335.

44 J. A. McCubrey, L. S. Steelman, W. H. Chappell, S. L. Abrams, G. Montalto, M. Cervello, F. Nicoletti, P. Fagone, G. Malaponte and M. C. Mazzarino, Oncotarget, 2012, 3, 954-987.

45 B. D. Manning and L. C. Cantley, Cell, 2007, 129, 1261-1274. 46 A. Dhanasekaran, S. Kotamraju, C. Karunakaran, S. V. Kalivendi, S. Thomas, J. Joseph and B. Kalyanaraman, Free Radical Biol. Med., 2005, 39, 567-583.

47 J.-Y. Yang, D. Michod, J. Walicki and C. Widmann, Biochem. Pharmacol., 2004, 68, 1027-1031.

48 G. Makin and C. Dive, Breast Cancer Res. Treat., 2001, 3, 150153.

49 T. Thalhamer, M. McGrath and M. Harnett, Rheumatology, 2008, 47, 409-414. 
50 Y. Zhang, X. Li, Z. Huang, W. Zheng, C. Fan and T. Chen, J. Nanomed. Nanotechnol., 2013, 9, 74-84.

51 V. Pierroz, T. Joshi, A. Leonidova, C. Mari, J. Schur, I. Ott, L. Spiccia, S. Ferrari and G. Gasser, J. Am. Chem. Soc., 2012, 134, 20376-20387.

52 W. Chen, N. Xu, L. Xu, L. Wang, Z. Li, W. Ma, Y. Zhu, C. Xu and N. A. Kotov, Macromol. Rapid Commun., 2010, 31, 228236.
53 L. Li, Y.-S. Wong, T. Chen, C. Fan and W. Zheng, Dalton Trans., 2012, 41, 1138-1141.

54 J. Su, H. Lai, J. Chen, L. Li, Y.-S. Wong, T. Chen and X. Li, PLoS One, 2013, 8, e63502.

55 J. Chen, Z. Luo, Z. Zhao, L. Xie, W. Zheng and T. Chen, Biomaterials, 2015, 71, 168-177. 